medRxiv preprint doi: https://doi.org/10.1101/2021.02.16.21251853; this version posted February 19, 2021. The copyright holder for this preprint

(which was not certified by peer review) is the author/funder, who has granted medRxiv a license to display the preprint in perpetuity.

It is made available under a CC-BY-NC-ND 4.0 International license .

\title{
Mortality in COVID-19 amongst women on Hormone Replacement Therapy or Combined Oral
} Contraception: A cohort study

Short title: COCP/HRT use in COVID-19 and mortality

Hajira Dambha-Miller ${ }^{1}$, William Hinton ${ }^{2}$, Mark Joy ${ }^{2}$, Michael Feher ${ }^{2}$ and Simon de Lusignan ${ }^{2,3}$

1. Primary Care Research Centre, University of Southampton

2. Nuffield Department of Primary Care Health Sciences, University of Oxford

3. Royal College of General Practitioners (RCGP) Research and Surveillance Centre (RSC), London, UK

Correspondence: Dr Hajira Dambha-Miller, Primary Care and Population Health, University of Southampton, Southampton, SO16 5ST, Email: H.Dambha-Miller@soton.ac.uk

\section{ABSTRACT}

Objective: To investigate the association between Hormone Replacement Therapy (HRT) or Combined Oral Contraception (COCP) use, and the likelihood of death in women with COVID-19.

Design: A cohort study

Setting: 465 general practices in England within the Oxford-Royal College of General Practitioners (RCGP) Research and Surveillance Centre (RSC) primary care database.

Population: 1,863,478 women aged over 18 years

Methods: We identified a cohort of women with COVID-19 from the computerised medical records of the RCGP RSC database. Mixed-effects logistic regression models were used to quantify the association between HRT or COCP use, and all-cause mortality among women with COVID-19 in unadjusted and adjusted models.

Results: There were 5451 COVID-19 cases within the cohort. HRT was associated with a significantly lower likelihood of all-cause mortality in COVID-19 (adjusted OR $0.22,95 \% \sqsupset \mathrm{Cl} 0.05$ to 0.94). There were no reported events for all-cause mortality in women prescribed COCPs. This prevented further examination of the impact of COCP.

Conclusions: Women on HRT with COVID-19 had a lower likelihood of death. Further work is needed in larger cohorts to examine the association of COCP in COVID-19. Our findings support the current hypothesis that oestrogens may contribute a protective effect against COVID-19 severity.

Funding: This study was funded by a School for Primary Care National Institute for Health Research grant (SPCR2014-10043).

Key words: HRT, COVID-19, mortality 


\section{INTRODUCTION}

The novel severe acute respiratory syndrome coronavirus 2 (SARS-CoV-2) continues to spread globally with males and females equally susceptible to the infection. However, males experience greater severity of infection with higher rates of hospitalisation and mortality.[1] A recent review of sex differences in COVID-19 including data from 38 countries reported mortality in males as 1.7 times higher than the average female. [2] Similar data have been observed in previous pandemics including the SARS-CoV (Severe Acute Respiratory Syndrome Corona Virus) and MERS-CoV (Middle East Respiratory Syndrome Corona Virus) outbreaks.[3] The reason for these sex differences is unclear. A range of hypotheses have been proposed from variations in patterned sex behaviours such as smoking, co-morbidities and sex-based immunological variations.[2] In particular, the role of oestrogen in female immune responses has received much attention.[4,5] Younger females or those with higher oestrogen levels are less likely to experience severe COVID-19 complications.[4] Earlier studies show that females mount faster and greater immune responses to viral infections through cellular and humoral immune responses.[2] Moreover, immune responses can be modulated by oestrogen through a reduction in T-cell exhaustion and suppression of IL-1 $\beta$ and IL-6 production.[6] This potentially limits the cytokine storm and subsequent respiratory failure that is characteristically triggered by SARS-CoV-2. This may explain why fewer women compared to men have been hospitalised, admitted to ITU or have died during the pandemic. [1]

Recent observational data suggests that women aged 18-45 years taking the COCP have a lower risk of COVID-19 infection $(P=<0.001)$ and a lower rate of hospital attendance $(P=0.023)$. $[7,8]$ Evidence on Hormone Replacement Therapy (HRT) has been more inconsistent.[7] To our knowledge, no studies have investigated the role of oestrogen containing products on mortality in COVID-19. The potential protective effects of oestrogen on the severity of COVID-19 has important public health and clinical relevance. In the absence of curative treatment for the infection, repurposing of existing drugs including exogenous oestrogen products requires rapid investigation. It is also necessary to understand the potential impact of these drugs for women taking them given public and prescriber concern. Accordingly, we quantified the association between COCP or HRT use, and the likelihood of mortality amongst females with COVID-19 during the first six months of the pandemic.

\section{METHODS}

\section{Study design, data source and population}

In this retrospective cohort study, we used the Oxford-Royal College of General Practitioners (RCGP) Research and Surveillance Centre (RSC) database of individual-level pseudonymised data that has been routinely collected from primary care records.[9] It includes continuous longitudinal data with sociodemographic information, prescribed medications, clinical diagnosis, symptoms, investigations and results. The database includes 465 GP practices in both rural and urban areas of England covering a nationally representative population of 3.7 million people. Within the database, we identified a cohort of women registered on the $1^{\text {st }}$ January 2020 who were aged over 18 years with confirmed or probable COVID-19. Confirmed cases were defined as those with a positive RT-PCR assay for SARS-CoV-2 on a nasal or pharyngeal swab and probable cases were those diagnosed radiologically or clinically based Public Health England's recommendations. Clinical symptoms included a new continuous cough, a fever $>37.8$ degrees or a loss/change in normal smell or taste. The variability in the availability of RT-PCR testing during the pandemic meant that most recorded cases in the dataset were diagnosed as probably cases. [10,11] Our previous work shows that clinical and probable cases are similar in terms of outcomes; for mortality, the odds ratios were $8.9(95 \% \mathrm{Cl}=$ 6.7 to $11.8, P<0.0001)$ and $9.7(95 \% \mathrm{Cl}=7.1$ to $13.2, P<0.0001)$ for RT-PCR confirmed and clinically diagnosed cases, respectively. [12] 
Exposure: HRT or COCP use

We defined the exposure as one or more HRT or COCP prescriptions within 6 months of a confirmed or suspected COVID-19 case. This had to be before case confirmation.

Outcome: All-cause mortality

The primary outcome was all-cause mortality during the follow-up period $\left(1^{\text {st }}\right.$ January to $21^{\text {st }}$ June 2020) as recorded in the electronic record.

Covariables:

We extracted data on age, ethnicity and socioeconomic status. Ethnicity was self-reported in the records.[13] For socioeconomic status, the English Index of Multiple Deprivation was used. [14,15] We combined IMD quintiles 1 and 2 because recent evidence shows that there is a low frequency of testing, leading to sparse data in the most deprived quintile.[14] We included the most recently available data on the household size as this is important in acquiring COVID-19 infection.[16] For clinical variables, we considered body mass index (BMI) as the most recent recording within the 12 months before the study start date and coding for co-morbidities were recorded as any history of hypertension, coronary heart disease, type 1 diabetes, type 2 diabetes, asthma, chronic obstructive pulmonary disease (COPD), and chronic kidney disease (CKD) stage 3-5. Smoking status categorised as non-smoker, active-smoker or ex-smoker. We also included prescriptions for prednisolone and/or disease-modifying anti-rheumatic drugs as a surrogate for immunosuppression. We used standardised coding required for NHS payment and administrative purposes to increase consistency and quality of data included.

\section{Statistical Analysis}

Sociodemographic and clinical characteristics were summarised using descriptive statistics, and we compared the characteristics of those with and without missing data. Univariable logistic regression models were used to quantify the association between HRT and COCP (separately) in relation to allcause mortality. We then ran multivariable models adjusting for age, sex, ethnicity, index of multiple deprivation, household size, BMI, and comorbidities. Mixed-effects models were performed to account for practice clustering. We ran a complete case analysis. Statistical analyses were performed using $R$ (Version 3.5.3). The level of significance was set at $5 \%$ and all statistical tests were 2-tailed. Model parameters were reported using odds ratios (ORs) and 95\% confidence intervals. Our findings are reported in line with the STROBE and RECORD guidelines for observational studies using routinely collected health data.

\section{Patient and public involvement}

Patients and members of the public contributed to the setting the research question, the outcome measures and the dissemination of our findings.

\section{Ethical approval}

This study received approval from the Oxford- RCGP RSC study approval committee (RSC_0920) and the University of Southampton Research Ethics committee (56309)

\section{RESULTS}

\section{Participant characteristics}

In this retrospective cohort study, the denominator population included 1,863,478 women across 465 general practices within the Oxford-RCGP RSC database during the first six months of the UK's COVID-19 pandemic. Within this sample, we identified a cohort of 5451 women who had COVID-19. 
The mean follow-up period was 164.9(SD 19.6) days. The mean age of the cohort was 59.0 years (SD 21.7); self-assigned ethnicity was predominantly White (64.8\%). There were 235 women with HRT prescriptions and 171 with a prescription for the COCP. Table 1 summarises sociodemographic and clinical characteristics in the whole cohort, and separated as those on HRT or COCP. During the follow up-period 664 (12.2\%) women died. Table 2 summarises the characteristics of women who died; they were more likely to be older with multiple morbidities.

\section{HRT use and all-cause mortality in COVID-19}

HRT use was associated with a lower likelihood of all-cause mortality in COVID-19 within unadjusted models (OR $0.15,95 \% \sqsupset \mathrm{Cl} 0.06$ to 0.37 ) and adjusted models (OR $0.22,95 \% \sqsupset \mathrm{Cl} 0.05$ to 0.94 ). We also observed that all-cause mortality risk was higher in COVID-19 amongst women who were older, underweight, from larger households, with hypertension, or on immunosuppressants. For those with asthma, this association seemed to be protective as women on HRT had a significantly lower odds of all-cause mortality (OR $0.58,95 \% \sqsupset \mathrm{Cl} 0.42$ to 0.81$)$. These results are shown in table 3 below.

\section{COCP use and all-cause mortality in COVID-19}

We had intended to examine COCP as an exposure but as there were no reported events for the outcome of interest (all-cause mortality) in women prescribed COCPs. Accordingly, we were unable to examine COCP use.

\section{DISCUSSION}

\section{Main findings}

In this cohort of 5451 women with COVID-19 who were followed up in the first six months of the pandemic, HRT use was associated with a lower likelihood of all-cause mortality.

\section{Strengths and limitation}

A major strength of this study is the use of a population-based cohort from 465 practices across England representing wide coverage with a denominator population of 3.6 million people. This included heterogeneity in sociodemographic and clinical variables. The data used is of high quality and completeness with twice-weekly updates that are also used by Public Health England to monitor the current and previous pandemics.[9] The availability of wide-ranging and precise data means that we were able to adjust for several confounders, although residual confounding is still possible. We considered both laboratory-confirmed and clinically probable cases as a single cohort due to the national inconsistency in testing availability. It is plausible that not all those with clinically probable cases had SARS-CoV-2. Recent work in Oxford RCGP database suggests that outcomes are similar in those with clinically probable and laboratory-confirmed cases.[17] Serology testing, if available nationally in the future could also be helpful. Our cohort is likely to reflect women with more severe COVID-19 symptoms who went for testing or made contact with a general practice for review. If asymptomatic or with milder symptoms, they may not have sought health advice and will not be captured in this cohort. In terms of the exposure, we examined medications based on prescriptions within the last 6 months rather than dispensed medications so there could be some overascertainment of exposure to oestrogens. Further, as oestrogen was highlighted as having a role in COVID-19 reasonably early in the pandemic, it is possible that some women may have stopped taking their medications before contracting the infection. Our study did not examine the type of preparation or dose of HRT. Nor did we investigate the duration of medication use and our follow-up period was short at less than six months. This might be important in oestrogen related immune 
responses where longer exposures to hormones could be significant.[2] We used all-cause mortality as our outcome, and some deaths may therefore be unrelated to COVID-19. There was substantial confusion about the classification of COVID-19 mortality in the early part of the pandemic including changes in Government guidance as the pandemic progressed. COVID-19 specific mortality was variable and as a new code, it may not have been widely used in primary care records. All-cause mortality is likely to be a more reliable measure especially in the early part of the pandemic in which our study is set.

\section{Interpretation}

To our knowledge, this is the first study to examine the use of exogenous oestrogens through HRT concerning all-cause mortality amongst women with COVID-19. Previous studies report lower rates of severe COVID-19 complications amongst women compared to men, and our findings give weight to current hypotheses suggesting that oestrogen may confer a protective effect against COVID-19. $[7,8]$ $[4,5]$ This is consistent with the findings of the COVID Symptom Study which is the largest observational study to date including 152,637 women for menopause status. [7] Their findings across the cohort suggests that higher oestrogen levels may protect against COVID-19. The mechanism to explain this may be through increased cellular and humoral immune responses in females with higher oestrogen levels. Recent evidence suggests that females have a higher level and faster generation of serum SARS-CoV-2 IgG antibody compared to males.[18] Higher oestrogen levels may also be able to better promote direct anti-viral activity of T-cells and modulate the uncontrolled immune response (cytokine storm) that has been observed in those with respiratory failure due to COVID-19. [4,5] Immune responses and oestrogen level decrease with age which might explain why previous studies and our results show a greater likelihood of worse outcomes in females with increasing age. $[1,7]$ However, amongst women on HRT with exogenous oestrogen the risk of all-cause mortality are reduced but still do not reach that of younger females presumably related to the direct effect of ageing on the immune system, and the increased number of morbidities acquired with age.[19] In the COVID Symptom Study described earlier, the association between HRT and COVID-19 hospitalisation in 17 798 women were variable and the authors do not show that HRT lowers the risk of hospitalisation but they do not report on mortality. [7] These differences might be explained by variations in HRT preparations, doses and duration which were not examined and as described above might be important in oestrogen led immune responses.[2] Other explanations may relate to differences in adjusted covariates which were limited to age, smoking and BMI in their study. As the pandemic progresses and a greater understanding of the virus emerges, it is necessary to consider additional covariates such as household size and co-morbidities which we included.[1] Our results show that increased age, co-morbidities, extreme BMI and immunosuppressants were all significantly associated with an increased likelihood of death amongst women with COVID-19; this is consistent with several recent reports.[20,21] There is some uncertainty in the literature about the role of asthma in the severity of COVID-19 outcome but we observed that being on HRT was associated with a significantly lower risk of mortality (OR $0.58,95 \% \sqsupset \mathrm{Cl} 0.42$ to 0.81$)$, suggesting that perhaps oestrogen is protective. However, these women with asthma are likely to also have been on asthma medication such as steroids which could contribute to some of the observed associations. [22]

\section{Conclusions}

We found that HRT use in COVID-19 infection was associated with a lower likelihood of death. Women on these medications should be encouraged to continue to take HRT during the pandemic. Further work is needed to explore the effect of variations in HRT doses, preparations and duration on COVID-19 complications. Additional research is also required in larger cohorts to examine the association been COCP and mortality in COVID-19.

\section{FUNDING:}


HDM is a National Institute for Health Research funded Academic Clinical Lecturer and has received NIHR SPCR funding for this project (SPCR2014-10043), and also acknowledges receipt of MRC funding for her COVID research (MR/V027778/1). The views and opinions expressed by authors in this publication are those of the authors and do not necessarily reflect those of the UK National Institute for Health Research (NIHR) or the Department of Health and Social Care

\section{AUTHOR CONTRIBUTION:}

HDM designed the study, wrote the first draft and edited subsequent versions of the manuscript. WH contributed to study design, led the data analysis and revised the manuscript. MJ provided advice on statistical methods. MF and Sdel contributed to the study design and revised the paper. Sdel also provided expertise on the RCGP RSC database.

\section{CONFLICT OF INTEREST STATEMENT:}

The authors declare that no support from any organisation and no financial relationships have influenced the submitted work. SdeL has had investigator led funding from industry, and is member of two advisory boards, all funding are via his University. No other authors have any competing interests to declare.

\section{ETHICAL APPROVAL:}

This study received approval from the Oxford- RCGP RSC study approval committee (RSC_0920) and the University of Southampton Research Ethics committee (56309) on the $6^{\text {th }}$ May 2020.

\section{ㄴIST OF TABLE LEGENDS:}

Table 1: Baseline characteristics of women with COVID-19 in the RCGP RSC database presented by those on HRT, COCP or neither drug

Table 2: Baseline characteristics of women with COVID-19 who died during the follow-up period in the Oxford-RCGP RSC database

Table 3: Association between HRT use and the likelihood of death in women with COVID-19 ( $n=$ 5451)

\section{REFERENCES:}

1 Williamson EJ, Walker AJ, Bhaskaran K, et al. Factors associated with COVID-19-related death using OpenSAFELY. Nature 2020;584. doi:10.1038/s41586-020-2521-4

2 Scully EP, Haverfield J, Ursin RL, et al. Considering how biological sex impacts immune responses and COVID-19 outcomes. Nature Reviews Immunology 2020;20. doi:10.1038/s41577-020-0348-8

$3 \quad$ Karlberg J, Chong DSY, Lai WYY. Do Men Have a Higher Case Fatality Rate of Severe Acute Respiratory Syndrome than Women Do? American Journal of Epidemiology 2004;159:229-31. doi:10.1093/aje/kwh056

Al-Lami RA, Urban RJ, Volpi E, et al. Sex Hormones and Novel Corona Virus Infectious Disease (COVID-19). Mayo Clinic Proceedings. 2020;95. doi:10.1016/j.mayocp.2020.05.013

5 Shabbir S, Hafeez A, Rafiq MA, et al. Estrogen shields women from COVID-19 complications by reducing ER stress. Medical Hypotheses 2020;143. doi:10.1016/j.mehy.2020.110148

6 Vaninov N. In the eye of the COVID-19 cytokine storm. Nature Reviews Immunology 2020;20. doi:10.1038/s41577-020-0305-6 

women from the COVID Symptom Study. medRxiv 2020;:2020.07.30.20164921. doi:10.1101/2020.07.30.20164921

Schroeder M, Jarczak D, Nierhaus A, et al. The majority of male patients with COVID-19 present low testosterone levels on admission to Intensive Care in Hamburg, Germany: a retrospective cohort study. doi:10.1101/2020.05.07.20073817

De Lusignan S, Correa A, Smith GE, et al. RCGP Research and Surveillance Centre: 50 years' surveillance of influenza, infections, and respiratory conditions. British Journal of General Practice. 2017;67:440-1. doi:10.3399/bjgp17X692645

10 Dambha-Miller H, Griffin SJ, Young D, et al. Posted on Annals of Family Medicine COVID-19 Collection, courtesy of Hajira Dambha-Miller. 2020. https://www. (accessed 7 May 2020).

11 de Lusignan S, Lopez Bernal J, Zambon M, et al. Emergence of a Novel Coronavirus (COVID19): A Protocol for Extending Surveillance Used by the Royal College of General Practitioners (RCGP) Research and Surveillance Centre (RSC) and Public Health England (PHE) (Preprint). JMIR Public Health and Surveillance 2020;6:e18606. doi:10.2196/18606

Joy M, Hobbs FR, Bernal JL, et al. Excess mortality in the first COVID pandemic peak: crosssectional analyses of the impact of age, sex, ethnicity, household size, and long-term conditions in people of known SARS-Cov-2 status in England. The British journal of general practice $\square$ : the journal of the Royal College of General Practitioners Published Online First: 19 October 2020. doi:10.3399/bjgp20X713393

13 Hippisley-Cox J, Coupland C, Vinogradova Y, et al. Predicting cardiovascular risk in England and Wales: Prospective derivation and validation of QRISK2. BMJ 2008;336:1475-82. doi:10.1136/bmj.39609.449676.25

14 Government D for C and L. The English Index of Multiple Deprivation (IMD) 2015-Guidance.

15 Tippu Z, Correa A, Liyanage $\mathrm{H}$, et al. Ethnicity recording in primary care computerised medical record systems: an ontological approach. Journal of Innovation in Health Informatics 2017;23. doi:10.14236/jhi.v23i4.920

16 Martin CA, Jenkins DR, Minhas JS, et al. Socio-demographic heterogeneity in the prevalence of COVID-19 during lockdown is associated with ethnicity and household size: Results from an observational cohort study. EClinicalMedicine 2020;25. doi:10.1016/j.eclinm.2020.100466

17 Joy M, Hobbs FR, Bernal JL, et al. Excess mortality in the first COVID pandemic peak: crosssectional analyses of the impact of age, sex, ethnicity, household size, and long-term conditions in people of known SARS-Cov-2 status in England. British Journal of General Practice 2020;70:bjgp20X713393. doi:10.3399/bjgp20x713393

18 Zeng F, Dai C, Cai P, et al. A comparison study of SARS-CoV-2 IgG antibody between male and female COVID-19 patients: A possible reason underlying different outcome between sex. Journal of Medical Virology 2020;92. doi:10.1002/jmv.25989

19 Koff WC, Williams MA. Covid-19 and Immunity in Aging Populations - A New Research Agenda. New England Journal of Medicine 2020;383:804-5. doi:10.1056/nejmp2006761

20 OpenSAFELY Collaborative T, Williamson E, Walker AJ, et al. OpenSAFELY: factors associated with COVID-19-related hospital death in the linked electronic health records of 17 million adult NHS patients. doi:10.1101/2020.05.06.20092999

21 Holman N, Knighton P, Kar P, et al. Risk factors for COVID-19-related mortality in people with type 1 and type 2 diabetes in England: a population-based cohort study. The Lancet Diabetes and Endocrinology 2020;8:823-33. doi:10.1016/S2213-8587(20)30271-0

22 Schultze A, Walker AJ, MacKenna B, et al. Risk of COVID-19-related death among patients with chronic obstructive pulmonary disease or asthma prescribed inhaled corticosteroids: an observational cohort study using the OpenSAFELY platform. The Lancet Respiratory Medicine 2020;8:1106-20. doi:10.1016/S2213-2600(20)30415-X 
Table 1: Baseline characteristics of women with COVID-19 in the RCGP RSC database presented by those on HRT, COCP or neither drug

\begin{tabular}{|c|c|c|c|c|}
\hline & $\begin{array}{c}\text { Total } \\
(\mathrm{N}=5451)\end{array}$ & $\begin{array}{l}\text { Neither drug } \\
(N=5045)\end{array}$ & $\begin{array}{c}\text { HRT } \\
(\mathrm{N}=235)\end{array}$ & $\begin{array}{c}\text { COCP } \\
(N=171)\end{array}$ \\
\hline \multicolumn{5}{|l|}{ Sociodemographic } \\
\hline Age (years) * & $59.0(21.7)$ & $60.2(21.7)$ & $54.6(9.4)$ & $29.3(7.4)$ \\
\hline Ethnicity recorded & $4356(79.9)$ & $4024(79.8)$ & $193(82.1)$ & $139(81.3)$ \\
\hline White & $3534(64.8)$ & $3231(64.0)$ & $179(76.2)$ & $124(72.5)$ \\
\hline Asian & $510(9.4)$ & 497 (9.9) & $6(2.6)$ & $7(4.1)$ \\
\hline Black & $211(3.9)$ & $203(4.0)$ & $4(1.7)$ & $4(2.3)$ \\
\hline Mixed \& other & $101(1.9)$ & $93(1.8)$ & $4(1.7)$ & $4(2.3)$ \\
\hline IMD Quintile recorded & $5326(97.7)$ & $4931(97.7)$ & $232(98.7)$ & $163(95.3)$ \\
\hline 5 (Least deprived) & $1136(20.8)$ & $1018(20.2)$ & $74(31.5)$ & $44(25.7)$ \\
\hline 4 & $1088(20.0)$ & $999(19.8)$ & $58(24.7)$ & $31(18.1)$ \\
\hline 3 & $1054(19.3)$ & 986 (19.5) & $36(15.3)$ & $32(18.7)$ \\
\hline $1 \& 2$ (Most deprived) & $2048(37.6)$ & $1928(38.2)$ & $64(27.2)$ & $56(32.7)$ \\
\hline $\begin{array}{l}\text { Settlement or } \\
\text { population density }\end{array}$ & $5328(97.7)$ & $4933(97.8)$ & $232(98.7)$ & $163(95.3)$ \\
\hline Rural & $933(17.1)$ & $833(16.5)$ & $65(27.7)$ & $35(20.5)$ \\
\hline Urban & $4395(80.6)$ & $4100(81.3)$ & $167(71.1)$ & $128(74.9)$ \\
\hline \multicolumn{5}{|l|}{ Clinical } \\
\hline BMI recorded & $5122(94.0)$ & $4724(93.6)$ & $231(98.3)$ & $167(97.7)$ \\
\hline $\operatorname{BMI}\left(\mathrm{kg} / \mathrm{m}^{2}\right)$ & $28.2(7.3)$ & $28.3(7.3)$ & $29.3(6.4)$ & $24.55(4.5)$ \\
\hline $\begin{array}{l}\text { Smoking status } \\
\text { recorded }\end{array}$ & $5328(97.7)$ & $4928(97.7)$ & $233(99.1)$ & $167(97.7)$ \\
\hline Non-smoker & $2128(39.0)$ & $1969(39.0)$ & $67(28.5)$ & $92(53.8)$ \\
\hline Active-smoker & $486(8.9)$ & $447(8.9)$ & $28(11.9)$ & $11(6.4)$ \\
\hline Ex-smoker & $2714(49.8)$ & $2512(49.8)$ & $138(58.7)$ & $64(37.4)$ \\
\hline Co-morbidity* & $3001(55.1)$ & $2859(56.7)$ & $116(49.4)$ & $26(15.2)$ \\
\hline All medications ${ }^{* *}$ & $2452(45.0)$ & $2333(46.2)$ & $100(42.6)$ & $19(11.1)$ \\
\hline
\end{tabular}

* Includes hypertension, coronary heart disease, diabetes (type 1 or type 2), chronic kidney disease stage 3-5, asthma, COPD, immunocompromised

** Includes antihypertensive medication, lipid-lowering medication, hypoglycaemic medication, inhalers, immunosuppressants 
Table 2: Baseline characteristics of women with COVID-19 who died during the follow-up period in the Oxford-RCGP RSC database

\begin{tabular}{|c|c|c|c|}
\hline & $\begin{array}{l}\text { Total } \\
\mathrm{N}=5451\end{array}$ & $\begin{array}{l}\text { Non-decedent } \\
\mathrm{N}=4787\end{array}$ & $\begin{array}{l}\text { Decedent } \\
N=664\end{array}$ \\
\hline Age (years) & $59.0(21.7)$ & $55.7(20.8)$ & $82.5(11.3)$ \\
\hline Ethnicity recorded & 4356 (79.9) & $3838(80.2)$ & $518(78.0)$ \\
\hline White & $3534(64.8)$ & $3064(64.0)$ & $470(70.8)$ \\
\hline Asian & $510(9.4)$ & $484(10.1)$ & $26(3.9)$ \\
\hline Black & $211(3.9)$ & $200(4.2)$ & $11(1.7)$ \\
\hline Mixed other & $101(1.9)$ & $90(1.9)$ & $11(1.7)$ \\
\hline $\begin{array}{l}\text { IMD Quintile } \\
\text { recorded }\end{array}$ & $5326(97.7)$ & $4671(97.6)$ & $655(98.6)$ \\
\hline 5 (Least deprived) & $1136(20.8)$ & $993(20.7)$ & $143(21.5)$ \\
\hline 4 & $1088(20.0)$ & $936(19.6)$ & $152(22.9)$ \\
\hline 3 & 1054 (19.3) & 918 (19.2) & $136(20.5)$ \\
\hline $\begin{array}{c}1 \& 2 \text { (Most } \\
\text { deprived) }\end{array}$ & 2048 (37.6) & $1824(38.1)$ & 224 (33.7) \\
\hline $\begin{array}{l}\text { Settlement or } \\
\text { population density }\end{array}$ & $5328(97.7)$ & 4671 (97.6) & 657 (98.9) \\
\hline Rural & $933(17.1)$ & $816(17.0)$ & $117(17.6)$ \\
\hline Urban & $4395(80.6)$ & $3855(80.5)$ & $540(81.3)$ \\
\hline BMI recorded & $5122(94.0)$ & & \\
\hline BMI $\left(\mathrm{kg} / \mathrm{m}^{2}\right)$ & $28.2(7.3)$ & $28.4(7.3)$ & $26.6(7.1)$ \\
\hline $\begin{array}{l}\text { Smoking status } \\
\text { recorded }\end{array}$ & 5328 (97.7) & $4684(97.8)$ & $26.6(7.1)$ \\
\hline Non-smoker & $2128(39.0)$ & 1912 (39.9) & $216(32.5)$ \\
\hline Active-smoker & $486(8.9)$ & $446(9.3)$ & $40(6.0)$ \\
\hline Ex-smoker & $2714(49.8)$ & $2326(48.6)$ & $388(58.4)$ \\
\hline Co-morbidity * & 3001 (55.1) & $2454(51.3)$ & $547(82.4)$ \\
\hline All medications ** & $2452(45.0)$ & $1980(41.4)$ & $472(71.1)$ \\
\hline
\end{tabular}

* Includes hypertension, coronary heart disease, diabetes (type 1 or type 2), chronic kidney disease stage 3-5, asthma, COPD, immunocompromised

** Includes antihypertensive medication, lipid lowering medication, hypoglycaemic medication, inhalers, immunosuppressants 
Table 3: Association between HRT use and the likelihood of death in women with COVID-19 $(n=5451)$

i) Unadjusted models

\begin{tabular}{|c|c|c|c|c|}
\hline & OR & \multicolumn{2}{|c|}{$95 \% \mathrm{Cl}$} & $\mathrm{p}$-value \\
\hline HRT use & 0.15 & 0.061 & 0.366 & 0.000 \\
\hline
\end{tabular}

\section{ii) Maximally adjusted models}

\begin{tabular}{|l|r|r|r|r|}
\hline & \multicolumn{1}{|c|}{ OR } & \multicolumn{2}{|c|}{$95 \% \mathrm{Cl}$} & p-value \\
\hline HRT use & 0.22 & 0.05 & 0.94 & 0.041 \\
\hline Age 40-64 (years) & 10.40 & 2.48 & 43.30 & 0.001 \\
\hline Age 65-74 (years) & 58.90 & 14.00 & 249.00 & 0.000 \\
\hline Age over 75+ & 123.00 & 29.70 & 514.00 & 0.000 \\
\hline Ethnicity Asian & 1.32 & 0.77 & 2.24 & 0.311 \\
\hline Ethnicity Black & 0.87 & 0.42 & 1.81 & 0.710 \\
\hline Ethnicity Mixed and other & 1.76 & 0.74 & 4.17 & 0.199 \\
\hline IMD Quintile 1 \& & 0.81 & 0.58 & 1.13 & 0.221 \\
\hline IMD Quintile 3 & 0.98 & 0.68 & 1.41 & 0.922 \\
\hline IMD Quintile 4 & 0.80 & 0.56 & 1.15 & 0.233 \\
\hline Household Size of 1 & 1.30 & 0.97 & 1.74 & 0.075 \\
\hline Household Size of 5-8 & 1.35 & 0.84 & 2.18 & 0.220 \\
\hline Household Size of >9 & 1.77 & 1.27 & 2.46 & 0.001 \\
\hline BMI Categorised as obese & 0.85 & 0.62 & 1.15 & 0.289 \\
\hline BMI Categorised as overweight & 0.87 & 0.66 & 1.16 & 0.350 \\
\hline BMI Categorised as underweight & 1.73 & 1.08 & 2.77 & 0.024 \\
\hline Active Smoker & 1.92 & 1.15 & 3.20 & 0.013 \\
\hline Ex-smoker & 1.21 & 0.94 & 1.56 & 0.144 \\
\hline Hypertension & 1.65 & 1.26 & 2.16 & 0.000 \\
\hline Coronary Heart Disease & 1.16 & 0.83 & 1.62 & 0.379 \\
\hline Type 1 Diabetes & 1.81 & 0.31 & 10.50 & 0.506 \\
\hline Type 2 Diabetes & 1.14 & 0.87 & 1.49 & 0.344 \\
\hline Chronic Kidney Disease Stage 3 to 5 & 1.18 & 0.91 & 1.52 & 0.215 \\
\hline Asthma & 0.58 & 0.42 & 0.81 & 0.001 \\
\hline COPD & 1.13 & 0.76 & 1.68 & 0.552 \\
\hline Immunosuppressants & 1.48 & 1.02 & 2.14 & 0.039 \\
\hline
\end{tabular}

The following reference categories were used: White for ethnicity, Age band: 18-39 years, IMD: IMD Quintile 5 (least deprived), Household size: 2-4 and for BMI category: Normal weight 
medRxiv preprint doi: https://doi.org/10.1101/2021.02.16.21251853; this version posted February 19, 2021. The copyright holder for this preprint (which was not certified by peer review) is the author/funder, who has granted medRxiv a license to display the preprint in perpetuity.

\author{
It is made available under a CC-BY-NC-ND 4.0 International license.
}

